\title{
PREVALENCIA DE Cryptosporidium Y Eimeria EN POTRILLOS DE CARRERA EN LA COSTA DEL DEPARTAMENTO DE LIMA
}

\author{
Miguel Ocampo V., ${ }^{1}$ Teresa López U. ${ }^{2}$, Armando González Z ${ }^{3}$ y \\ Marcos Copaira M. ${ }^{4}$
}

\section{Ninewist}

The aim of the study was to determine the prevalence of Cryptosporidium and Eimeria in thoroughbred foals from Lima. During the months from August through December 1998, a total of 369 fecal samples were collected at several breeding centers from suckling foals aged 0 to 52 weeks. Direct fresh smears were fixed with methanol and processed using modified Zielh Neelsen stain for cryptosporidia diagnosis. For Eimeria, the samples were concentrated using Formalin-ether sedimentation and examined microscopically. Cryptosporidium prevalence was $48 \pm 5 \%(176 / 369)$ with a corrected value of $55 \pm 5 \%$, and statistically significant differences were found among animals belonging to different age groups $(\mathrm{P}<0.001)$. Six out of the 369 samples examined for Eimeria were positive $(2 \pm 1 \%)$. None of the Cryptosporidium or Eimeria positive foals had diarrhea, and the two foals with diarrhea tested negative to both protozoa.

Key words: Cryptosporidium, cryptosporidiosis, Eimeria, eimeriosis, equines.

\section{Rrownth}

Se determinó la prevalencia de Cryptosporidium y Eimeria en muestras fecales $(n=369)$ recolectadas de potrillos de carrera de 0 hasta 52 semanas de edad, en diversos centros de crianza localizados en la costa del departamento de Lima, durante el período de agosto a diciembre de 1998. Un frotis de heces frescas fue fijado con metanol y teñidos con la técnica de Ziehl Neelsen Modificado para el diagnóstico de criptosporidia. Para el diagnóstico de Eimeria las muestras fueron concentradas usando la técnica de sedimentación en formol-éter, luego el sedimento fue examinado directamente para detectar a este parásito. La prevalencia de Cryptosporidium fue de $48 \pm 5 \%$ (176/369), con una prevalencia corregida de $55 \pm 5 \%$, pudiéndose observar diferencias significativas entre los diferentes grupos etáreos $(\mathrm{P}<0.001)$. Seis de las 369 muestras examinadas para Eimeria fueron positivas $(2 \pm 1 \%$ ). Ninguno de los potrillos positivos a Cryptosporidium o Eimeria presentaban diarrea. Sin embargo, los dos potrillos en los que se observó diarrea fueron negativos a ambos protozoarios.

Palabras claves: Cryptosporidium, criptosporidiosis, Eimeria, eimeriosis, equinos.

\footnotetext{
'Práctica Privada

${ }^{2}$ Laboratorio de Parasitología-FMV-UNMSM

${ }^{3}$ Medicina Veterinaria Preventiva-FMV-UNMSM

+ Salud Animal y Salud Pública-FMV-UNMSM
} 


\section{Introtiogth}

El Cryptosporidium y la Eimeria son parásitos protozoarios que han resultado ser de significativa importancia en los equinos por ser causantes de una tasa de morbilidad relativamente alta en potrillos en diversos países y además, han sido descritas como causantes de infección en diferentes especies animales, entre mamiferos domésticos y silvestres, aves, reptiles e incluso el hombre (Anderson, 1982; Tzipori, 1983; Chermette \& Boufassa, 1988; Angus, 1990).

La criptosporidiosis en equinos ha sido reportado en muchas partes del mundo (Tzipori, 1983; Xiao \& Herd, 1994a) y ha sido asociado con la ocurrencia de diarrea en potrillos inmunodeficientes e inmunocompetentes (Snyder et al., 1978; Gibson et al., 1983; Coleman et al., 1989; Mair et al., 1990) además, de constituirse en un problema zoonótico (Johnson et al., 1997). La prevalencia de infección por Eimeria ha sido reportado en diversos países siendo esta muy variable (Mc Queary et al., 1977; Lyons et al., 1988; Beelitz et al., 1994) y su rol en la producción de diarrea en equinos no es muy clara.

El presente estudio ha sido diseñado para investigar la prevalencia de Cryptosporidium y Eimeria en potrillos de carrera, de diferentes grupos etáreos, en la costa del departamento de Lima.

\section{Merewales Mrowas}

\section{Lugar de estudio y animales}

Durante los meses de agosto a diciembre de 1998, se procedió al muestreo de 14 haras de caballos de carrera localizados: 6 en la zona sur (Cañete), 6 en la zona Norte (Sayán) y 2 en la zona centro (Chaclacayo) de la costa del departamento de Lima. Se recolectaron muestras fecales de 369 potrillos de carrera cuyas edades fluctuaban entre las
0 a 52 semanas, las muestras fueron tomadas directamente del recto o frescas recientemente excretadas; posteriormente, fueron transportadas al laboratorio en bolsas plásticas individualizadas e identificadas y se conservaron en refrigeración $\left(4^{\circ} \mathrm{C}\right)$ para luego realizar el examen parasitológico.

\section{Identificación del parasito}

Para el diagnóstico de ooquistes de Cryptosporidium se utilizó la tinción de Ziehl Neelsen Modificado (Henricksen y Pottlenz, 1981); para el cual se realizó un frotis de heces en un portaobjeto y se fijó luego en metanol por 5 minutos, el frotis luego se coloreó con fucsina fenicada por 20 minutos, se lavó y se decoloró con ácido sulfúrico al $2 \%$ por 20 segundos, luego de lavar se contrastó con verde malaquita $5 \%$ por 5 minutos, se lavó, secó y se observó al microscopio con objetivo $40 \mathrm{X}$ y $100 \mathrm{X}$.

Para el diagnóstico de Eimeria se utilizó un método cualitativo de sedimentación en formol-éter (Ritchie) y un método cuantitativo de Mc Master Modificado, para realizar el recuento de ooquistes que hubieran resultado positivos al primer examen.

Para la sedimentación en formol-éter, se colocó en primer lugar una pequeña muestra de heces en un recipiente con $10 \mathrm{ml}$ de formol al $10 \%$ y se dejó por 30 minutos, posteriormente, se tamizó en un tubo y se centrifugó a $1500 \mathrm{rpm}$ por 5 minutos, se decantó luego el sobrenadante y el sedimento se resuspendió con formol y éter $(7$ y $3 \mathrm{ml}$, respectivamente), se tapó y agitó el tubo hasta mezclar completamente, y se centrifugó a $1500 \mathrm{rpm}$ por 5 minutos, después del cual se formaron 4 capas decantando las 3 capas superiores, dejando el sedimento, el cual en un portaobjeto fue observado al microscopio a $10 \mathrm{X}$ y $40 \mathrm{X}$ para buscar ooquistes de Eimeria.

Para el método de Mc Master Modificado se homogeneizó $3 \mathrm{~g}$ de heces en $42 \mathrm{ml}$ de agua, se tamizó y el filtrado se llenó en un tubo de $15 \mathrm{ml}$, luego de sedimentar se eliminó 
el sobrenadante y se reemplazó con una solución salina $(\mathrm{CINa})$, se homogeneizó, se tomó una muestra con un gotero y se llenó la cámara de Mc Master, y se procedió al conteo de los ooquistes en el microscopio.

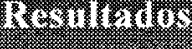

\section{Cryptosporidium:}

De las 369 muestra analizadas, 176 resultaron positivos a ooquistes de Cryptosporidium parvum, lo que representa una prevalencia de $48 \pm 5 \%$, y una prevalencia corregida de $55 \pm 5 \%$. (Cuadro 1 ).
En el Cuadro 2, se observa la distribución de animales positivos a Cryptosporidium de acuerdo a edad. Se distingue una mayor frecuencia en potrillos de 0 a 8 semanas de edad, existiendo una tendencia al descenso de animales positivos conforme avanza la edad, siendo estadísticamente significativo $(P<0.001)$ en agrupaciones por rangos de edad de 8 semanas.

La prevalencia de potrillo positivos según zona de muestreo, fue de $58 \pm 7 \%$ en la zona sur; $51 \pm 8 \%$ en el norte y $55 \pm 14 \%$ en la zona centro; no observándose diferencias significativas $(\mathrm{P}>0.05)$

Cuadro 1. Prevalencia corregida ( \pm I.C.) en potrillos de carrera infectados por Cryptosporidium en la costa del Departamento de Lima (agosto a diciembre, 1998)

\begin{tabular}{lcccc}
\hline Condición & $\begin{array}{c}\text { Muestras } \\
\mathbf{n}\end{array}$ & $\begin{array}{c}\text { Positivos } \\
\mathbf{n}\end{array}$ & $\begin{array}{c}\text { Porcentaje } \\
(\%)\end{array}$ & $\begin{array}{c}\text { Prevalencia } \\
\text { Corregida }\end{array}$ \\
\hline Diarreicos & 2 & 0 & 0 & 0 \\
Sanos & 367 & 176 & 48 & $55 \pm 5 \%$ \\
\hline Total & 369 & 176 & 48 & $55 \pm 5 \%$ \\
\hline
\end{tabular}

Cuadro 2. Prevalencia corregida ( \pm I.C.) de Cryptosporidium según estrato etáreo en potrillos de carrera en la costa del Departamento de Lima (agosto a diciembre, 1998).

\begin{tabular}{ccccc}
\hline $\begin{array}{c}\text { Edad } \\
\text { ( Semanas })\end{array}$ & $\begin{array}{c}\text { Muestras } \\
\mathrm{n}\end{array}$ & $\begin{array}{c}\text { Positivos } \\
\mathrm{n}\end{array}$ & $\begin{array}{c}\text { Porcentaje } \\
(\%)\end{array}$ & $\begin{array}{c}\text { Prevalencia } \\
\text { Corregida }\end{array}$ \\
\hline $0-8$ & 54 & 39 & 72 & $83 \pm 10 \%$ \\
$9-16$ & 27 & 17 & 63 & $72 \pm 17 \%$ \\
$17-24$ & 111 & 54 & 49 & $56 \pm 9 \%$ \\
$25-32$ & 69 & 29 & 42 & $48 \pm 12 \%$ \\
$33-40$ & 50 & 20 & 40 & $46 \pm 14 \%$ \\
$41-52$ & 58 & 17 & 29 & $34 \pm 12 \%$ \\
\hline Total & 369 & 176 & 48 & $55 \pm 5 \%$ \\
\hline
\end{tabular}

$\mathrm{X}^{2} 0.001,5$ g. $1 .=25.51^{* *}$ 
Cuadro 3. Prevalencia de potrillos de carrera infectados por Eimeria en la costa del Departamento de Lima (agosto a diciembre, 1998)

\begin{tabular}{lccc}
\hline Condición & $\begin{array}{c}\text { Muestras } \\
\mathbf{n}\end{array}$ & $\begin{array}{c}\text { Positivos } \\
\mathbf{n}\end{array}$ & Prevalencia \\
\hline Diarreicos & 2 & 0 & 0 \\
Sanos & 367 & 6 & $2 \pm 1 \%$ \\
\hline Total & 369 & 6 & $2 \pm 1 \%$ \\
\hline
\end{tabular}

\section{Eimeria:}

Mediante el método de sedimentación en formol éter y reevaluado por el método de flotación en solución salina, se obtuvo 6 muestras positivas a Eimeria sp. (Cuadro 3) lo que representa el $2 \%$ del total de animales muestreados, con una frecuencia de presentación de $2 \pm 1 \%$ (I.C. 95\%).

El Cuadro 4, muestra la distribución de animales positivos a Eimeria de acuerdo a la edad. Se observa que los 6 potrillos positivos oscilan entre las 9 a 32 semanas de edad; siendo mayor la frecuencia entre los de 25 a 32 semanas en donde se halló 4 animales infectados $(6 \pm 6 \%)$, y entre las 9 a 16 semanas y, entre las 17 a 24 semanas un animal positivo en cada caso, hallándose una frecuencia de $4 \pm 7 \%$ y $1 \pm 2 \%$, respectivamente.

Además por el método de Mc Master se logró obtener un rango de 50-150 ooquistes de Eimeria sp. por gramo de heces en los potrillos examinados.

\section{Disematom}

Los resultados de este estudio han confirmado que la infección por $C$. parvum es común en los potrillos (Tzipori, 1985), con una prevalencia mayor que los reportados en otras partes del mundo (Chermette $e t a l$, 1987; Xiao \& Herd, 1994b), aunque algunos autores han reportado la presencia de la

Cuadro 4. Prevalencia de Eimeria según estrato etáreo en potrillos de carrera en la costa del Departamento de Lima (agosto a diciembre, 1998)

\begin{tabular}{cccc}
\hline $\begin{array}{c}\text { Edad } \\
\text { (Semanas) }\end{array}$ & $\begin{array}{c}\text { Muestras } \\
\mathrm{n}\end{array}$ & $\begin{array}{c}\text { Positivos } \\
\mathrm{n}\end{array}$ & Prevalencia \\
\hline $0-8$ & 54 & 0 & 0 \\
$9-16$ & 27 & 1 & $4 \pm 7 \%$ \\
$17-24$ & 111 & 1 & $1 \pm 2 \%$ \\
$25-32$ & 69 & 4 & $6 \pm 6 \%$ \\
$33-40$ & 50 & 0 & 0 \\
$41-52$ & 58 & 0 & 0 \\
\hline Total & 369 & 6 & $2 \pm 1 \%$ \\
\hline
\end{tabular}


infección en más del $90 \%$ de los potrillos y caballos adultos estudiados, sugiriendo un alto rango de exposición de los equinos a este parásito (Tzipori \& Campbell, 1981)(Coleman et al., 1989).

Esta alta prevalencia de la infección pudo haber sido influenciada por varios factores como: incremento de animales destetados (mayores de 5 meses) positivos a C. parvum, aumentando el riesgo de contaminación de los pastos y la infección a potrillos más jóvenes, temperaturas adecuadas durante el año que puede haber contribuido a una mejor esporulación de los ooquistes diseminados en el medio, diferencias en el manejo de los haras comparada con otros países, y el uso constante de los mismos potreros lo que permite una mayor acumulación del parásito en los pastizales.

No se encontró asociación entre animales positivos a criptosporidiosis en animales aparentemente sanos $(48 \%)$ versus potrillos con diarrea $(0 \%)$. Estos resultados confirman estudios anteriores donde se hallaron rangos de 2.4 a $60 \%$ de potrillos aparentemente normales infectados con el parásito sin signos de diarrea (Xiao \& Herd, 1994a).

Los efectos patogénicos del Cryptosporidium aún no están claros, algunos autores aseguran que la presencia de signos clínicos tiene que ver con la intensidad de la infección (Xiao \& Herd, 1994 a), mientras que otros señalan que una inmunodepresión es necesaria para la presencia de cuadros de diarrea en equinos (Gibson et al., 1983; Mair et al., 1990).

Los animales positivos fueron encontrados en todos los grupos etáreos estudiados. La mayor prevalencia fue registrada entre las 0 a 8 semana de edad y la infección ya estaba presente en potrillos menores de 4 semanas como fue reportado anteriormente en equinos (Xiao \& Herd, 1994 a) y en rumiantes (Olson et al., 1997), lo que podría deberse al período pre-patente del parásito
(4-5 días) (Anderson, 1982); susceptibilidad debido al poco desarrollo inmunológico (Tzipori, 1983) y/o al contacto que tienen los neonatos con potrillos infectados $y$, posiblemente con madres infectadas subclínicamente. (Coleman et al., 1989; Xiao \& Herd, 1994b).

A medida que la edad avanza se va desarrollando el sistema inmunológico (Rose, 1986), por lo que el número de animales positivos a Cryptosporidium va disminuyendo; además otros mecanismos no específicos como las barreras fisiológicas del intestino (Tzipori, 1985) y la presencia de una adecuada flora intestinal (Harp et al., 1992) pueden estar involucrados en la resistencia a la infección aparte del sistema inmune.

En el presente trabajo se pudo observar que potrillos de hasta 52 semanas de edad presentaban la infección, tal como indican Chermette et al. (1987), Xiao \& Herd (1994) y Lengronne et al.(1985) quienes reportaron potrillos de hasta 10 meses positivos a $C$. parvum, presentando algunos de ellos diarrea. La presentación de animales positivos en estas edades puede estar influenciada por infecciones tardias (Soulsby, 1987) o por el factor autoinfección (Fayer \& Ungar, 1986), que sería la causa de largos períodos de excreción de ooquistes tal como fue reportado en gatos experimentalmente infectados (Asahi et al., 1991).

Mediante el método de sedimentación en formol-éter y por el método de Mc Master Modificado, se encontró $6(2 \pm 1 \%)$ potrillos positivos a Eimeria $s p$. El parásito observado resultó ser una Eimeria sp., coccidia del cual se desconoce su ciclo de desarrollo, patogenicidad y prevalencia (Soulsby, 1987). Sólo un estudio realizado tiempo atrás, reporta la presencia de Eimeria $s p$. en equinos de Lima (Chávez \& Guerrero, 1960). La $E$. leuckarti, coccidia ampliamente distribuida en el mundo con prevalencias de 40 a $80 \%$ (Lyons et al., 1988; Beelitz et al., 1994), no pudo ser observada lo que sugiere la no existencia de 
este parásito dentro de la zona de muestreo o estaría muy por debajo de los límites registrados en otros estudios (Lyons et al., 1988).

De los seis potrillos positivos a Eimeria $s p$. ninguno presentaba cuadro de diarrea, to que sugiere la no patogenicidad de este parásito o que la dosis infectante no sea la adecuada para causar enfermedad; algo similar que en la $E$. leuckarti, donde diversos estudios demuestran una falla de asociación entre la infección por Eimeria y la presencia de diarrea (Bauer, 1988; Lyons et al., 1988; Beelitz et al., 1994).

Los potrillos infectados con Eimeria $s p$. se encontraban dentro de las 9 a 32 semanas de edad, observándose la mayor frecuencia entre las 25 a 32 semanas, lo que coincide con estudios en E. leuckarti donde se hallaron animales positivos en edades avanzadas (Sutoh et al., 1976; Mc Queary et al., 1977; Lyons et al., 1988). Estos mismos trabajos sugieren que la eimeriosis a esta edad puede deberse a una infección tardía muy común en infecciones naturales o a una excreción intermitente con intervalos negativos a ooquistes.

\section{Cmominnts}

1. El parásito Cryptosporidium parvim es común en potrillos en la Costa del Departamento de Lima.

2. La Eimeria sp. tuvo baja prevalencia en los potrillos muestreadas. El parásito observado fue diferente a la E. leuckarti.

\section{Whatrit artwh}

1. Anderson, B.C. 1982. Cryptosporidiosis: A review. J. Am. Vet. Med. Assoc., 180: 1455-1456.

2. Angus, K.W. 1990. Cryptosporidiosis in rumiants. In: Cryptosporidiosis in man and animals (Eds. Dubey, J.P.; Fayer, R. \& Speer, C.A.) C.R.C. Press, Boca Ratón, p.p. 83-105.

3. Asahi, H.; T. Koyama; H. Arai; Y. Funakoshi; H. Yamaura; $R$. Shirasaka y K. Okutomi. 1991. Biological nature of Cryptosporidium $s p$. isolated from a cat. Parasitol. Res., 77: 237-240.

4. Bauer, C. 1988. Prevalence de Eimeria leuckarti (Flesh, 1883) and intensity of faecal oocyst output in a herd of horses during a summer grazing season. Vet. Parasitol., 30: 11-15.

5. Beelitz, P.; N. Rieder y R. Gothe. 1994. Eimeria leuckarti infections in foals and their mothers in upper Bavaria. Tieraztl. Prax., 22: 377-381.

6. Chavez, C. y C. Guerrero. 1960. Ecto y endoparásitos identificados en el Departamento de Parasitología de la Facultad de Medicina Veterinaria (19471960). Revista de la Facultad de Medicina Veterinaria, 15: 48-68.

7. Chermette, R.; S. Boufassa; C. Soule; C. Tarnau; O. Couderc y D. Lengronne. 1987. La cryptosporidiose equine: une parasitose meconnue. Bulletin CEREOPA, 13e Journee d'Etudes. Paris, p.p. 81-94.

8. Chermette, R. y S. Boufassa. 1988. Cryptosporidiosis: a cosmopolitan disease in animals and in man. O.I.E., Tech. Series $N^{\circ} 5$, Paris.

9. Coleman, S.V.; T.R. Klei; D.D. French; M.R. Chapman y R.E. Corstuet. 1989. Prevalence of Cryptosporidium sp. in equids in Louisiana. Am. J. Vet. Res., 50: 575-577.

10. Fayer, R. y L.P. Ungar. 1986. Cryptosporidium spp. and Cryptosporidiosis. Microbiol. Rev., 50: 458-483.

11. Gibson, J.A.; M.W. Hill y M.J. Huber. 1983. Cryptosporidiosis in Arabian foals with severe combined inmunodeficiency. Aust.Vet. J., 60: 378-379.

12. Harp, J.A.; W. Chen y A.G Harmsen. 1992. Resistance of severe combined inmunodeficient mice toinfection with 
Cryptosporidium parvum: The importance of intestinal microflora. Infect. Immun., 60: 3509-3512.

13. Henricksen, S. y J. Pottlenz. 1981. Staining of cryptosporidia by a modified Zielh Neelsen technique. Acta Vet. Scand., 22: 594-596.

14. Johnson, E.; E.R. Atwill; M.E. Filkins y J. Kahosh. 1997. The prevalence of shedding of Cryptosporidium and Giardia spp. Based on a single fecal sample collection from each of 91 horses used for backcountry recreation. J. Vet. Diagn. Invest., 9: 56-60.

15. Lengronne, D.; G Reigner; P. Veau; $R$. Chermette; S. Boufassa y C. Soule. 1985. Cryptosporidiose chez des poulains diarrheiques. Poini. Vet., 17: 528-529.

16. Lyons, E.T.; J.H. Drudge y S.C. Tolliver. 1988. Natural infection wih Eimeria leuckarti: Prevalence of oocysts in feces of horses foals on several farms in Kentucky during 1986. Am. J. Vet. Res., 49: 96-98.

17. Mair, T.S.; F.G Taylor; D.A. Harbour y G.R. Pearson. 1990. Concurrent Cryptosporidium and Coronavirus infections in an Arabian foal with combined inmunodeficiency syndrome. Vet. Rec., 126: 127-130.

18. Mc Queary, C.A.; D.E. Worley y J.E. Catlin. 1977. Observations on the life cycle and prevalence of Eimeria leuckarti in horses in Montana. Am. J. Vet. Res., 38: 1673-1674.
19. Olson, M.E.; N.J. Guselle; R.M. O'Handley; M.L. Swift; T.A. Mc Allister; M.D. Jelinski y D.W. Morck. 1997. Giardia and Cryptosporidium in dairy calves in British Columbia. Can. Vet. J., 38: 703-706.

20. Snyder, S.P.; J.J. England y A.E. Mc Chesney. 1978. Cryptosporidiosis in inmunodeficient Arabian foals. Vet. Pathol., 15: 12-17.

21. Soulsby, E.J. 1987. Parasitología y enfermedades parasitarias de los animales domésticos. $7 \mathrm{ma}$. Ed., p. 610615. México: Interamericana S.A..

22. Sutoh, M.; Y. Saheki; R. Ishitani; S. Inui; M. Narita; H. Hamazaki y T. Yokota. 1976. Eimeria leuckarti infection in foals. Natl. Inst. Anim. Healthy, 16: 59-64.

23. Tzipori, S. y I. Campbell. 1981. Prevalence of Cryptosporidium antibodies in 10 animals species. J. Clin. Microbiol., 14: 455-456.

24. Tzipori, S. 1983. Cryptosporidiosis in animals and in humans. Microbiol. Rev., 47: 84-96.

25. Tzipori, S. 1985. Cryptosporidium: Notes on epidemiology and pathogenesis. Parasitol. Today, 1: 159-165.

26. Xiao, L. y R.P. Herd. 1994a. Review of equine Cryptosporidium infection. Equine Vet. J., 26: 9-13.

27. Xiao, $L$ y R.P. Herd. 1994b. Epidemiology of equine Cryptosporidium and Giardia infections. Equine Vet. J., 26: 14-17. 